\title{
Identification of fall predictors in the active elderly population from the routine medical records of general practitioners
}

\author{
Vieri Lastrucci ${ }^{1}$, Chiara Lorini ${ }^{2}$, Giada Rinaldi ${ }^{3}$ and Guglielmo Bonaccorsi ${ }^{4}$ \\ ${ }^{1}$ Research Fellow, Dipartimento di Scienze della Salute, Università degli Studi di Firenze, Florence, Italy \\ ${ }^{2}$ Researcher, Dipartimento di Scienze della Salute, Università degli Studi di Firenze, Florence, Italy \\ ${ }^{3}$ General Practitioner, Dipartimento di Medicina Sperimentale e Clinica, Università degli Studi di Firenze, Florence, Italy \\ ${ }^{4}$ Associate Professor, Dipartimento di Medicina Sperimentale e Clinica, Università degli Studi di Firenze, Florence, Italy
}

\begin{abstract}
Aim: To evaluate the possibility of determining predictors of falls in the active communitydwelling elderly from the routine medical records of the general practitioners (GPs). Background: Time constraints and competing demands in the clinical encounters frequently undermine fall-risk evaluation. In the context of proactive primary healthcare, quick, and efficient tools for a preliminary fall-risk assessment are needed in order to overcome these barriers. Methods: The study included 1220 subjects of 65 years of age or older. Data were extracted from the GPs' patient records. For each subject, the following variables were considered: age, gender, diseases, and pharmacotherapy. Univariate and multivariable analyses have been conducted to identify the independent predictors of falls. Findings: The mean age of the study population was $77.8 \pm 8.7$ years for women and $74.9 \pm 7.3$ years for men. Of the sample, $11.6 \%$ had experienced one or more falls in the previous year. The risk of falling was found to increase significantly $(P<0.05)$ with age $(\mathrm{OR}=1.03 ; 95 \% \mathrm{Cl}=1.01-1.05)$, generalized osteoarthritis $(\mathrm{OR}=2.01 ; 95 \% \mathrm{Cl}=1.23-3.30)$, tinnitus $(\mathrm{OR}=4.14 ; 95 \%$ $\mathrm{Cl}=1.25-13.74)$, cognitive impairment $(\mathrm{OR}=4.12 ; 95 \% \mathrm{Cl}=2.18-7.80)$, and two or more co-existing diseases ( $\mathrm{OR}=5.4 ; 95 \% \mathrm{Cl}=1.68-17.39$ ). Results suggest that it is possible to identify patients at higher risk of falling by going through the current medical records, without adding extra workload on the health personnel. In the context of proactive primary healthcare, the analysis of fall predictors from routine medical records may allow the identification of which of the several known and hypothesized risk factors may be more relevant for developing quick and efficient tools for a preliminary fall-risk assessment.
\end{abstract}

Key words: accidental falls; aged; independent living; proactive primary healthcare; risk factors; routine medical records

Received 22 September 2016; revised 14 July 2017; accepted 27 July 2017; first published online 5 September 2017

\section{Introduction}

Falls are one of the most common causes of injury, loss of function, and death among the elderly population (Fried et al., 2004). Every year, about

Correspondence to: Vieri Lastrucci, Dipartimento di Scienze della Salute, Università degli Studi di Firenze, Viale GB Morgagni 48, Florence, 50134 Italy. Email: vieri.lastrucci@ gmail.com
$30 \%$ of the population over 65 years of age are victim of falls (Todd and Skelton, 2004). However, since only less than half of the falls are reported to physicians or healthcare professionals, it is not easy to provide an accurate estimate of the true incidence (Stevens et al., 2012). Elderly people are more susceptible to falls and injuries due to the higher prevalence of clinical diseases, age-related physiological changes, and delayed functional recovery. 
Approximately $10 \%$ of falls are reported to cause serious damages - especially head trauma or hip fracture (Goldacre et al., 2002; Rubenstein and Josephson, 2002). Furthermore, the psychological consequences of a fall should be taken into account, as fear of falling could lead to severe restriction in the activities of daily living, leading to depression, social isolation, and loss of muscle tone, which in turn results in a vicious circle that could lead to an additional risk of falling in the long run (Murphy and Isaacs, 1982; Zijlstra et al., 2007).

A fall is a multi-factorial event in which there is a concurrence of intrinsic factors related to the characteristics of the person - represented by agerelated physiological changes, comorbidities, and drug therapy - and extrinsic factors related to the environmental risks (Lord et al., 2006; Rubenstein, 2006). Although the most significant factors for young people are related to the environment, the role of intrinsic risk factors becomes increasingly relevant with age. In the literature, more than 400 different risk factors related to falls are described. Of these, the most relevant ones are impaired balance, dizziness/vertigo, visual or musculoskeletal disorder, cognitive impairment, neurological diseases, cardiovascular diseases, and medications such as psychotropic drugs (Rubenstein, 2006). Several studies have shown that the risk of falling increases dramatically with the increased presence of risk factors (Faulkner et al., 2009; Shumway-Cook et al., 2009; Sibley et al., 2014).

Adequate medical and environmental evaluation and treatment for the underlying medical risk factors could prevent many falls and adverse outcomes that may force an elderly person into a vicious cycle of loss of functionality and autonomy, which increases the fragility and the need for assistance. Despite the existence of guidelines, clinical pathways, and tools for falls prevention, time constraints and competing demands in the clinical environment frequently undermine fallrisk evaluation and management (Wenger et al., 2003; Tinetti et al., 2006). In a community setting especially in the context of proactive primary healthcare - quick and efficient tools for a preliminary fall-risk assessment based on routine medical records could help to overcome these barriers and to develop tailored and cost-effective prevention programmes. The objective of the present study is to evaluate the possibility of determining fall

Primary Health Care Research \& Development 2018; 19: 131-139 predictors in the active community-dwelling elderly from the routine medical records of the general practitioners (GPs).

\section{Methods}

The study was approved by the local Ethics Committee and was conducted according to the principles described in the Declaration of Helsinki.

The study was conducted in a rural municipality of about 10000 inhabitants located in central Italy. All the GPs $(n=4)$ at a Community Health Center (Unità Complessa di Cure Primarie) serving the municipality were recruited for the study. According to the regulation of the National Healthcare Systems, in Italy every citizen and foreign resident above the age of 18 is entitled to be registered as a patient with a GP. The healthcare provided from GPs is free of charge for all patients.

Data were extracted from the GPs' routine medical records of the patients through the software Millewin ${ }^{\circledR}$ - an ad hoc software developed for and used by a majority of Italian GPs. Records were extracted and reviewed between January and March 2016 and the data of patients aged 65 years and older were included in the study. In order to select an active elderly population, bedridden or non-ambulatory patients and patients with severe dementia were excluded from the study.

For each subject, the following variables were considered: history of falls in the previous year, age, gender, past medical history of diseases, and current pharmacotherapy. In patients with more than one fall event, only the first event was considered in the analysis. The Millewin software uses the ICDIX$\mathrm{CM}$ classification and the anatomical therapeutic chemical (ATC) classification system to classify diseases and pharmacotherapy, respectively.

The diseases were analysed as a dichotomous variable - either individually (in terms of the presence or absence of a specific disease) or as a group (in terms of the presence or absence of at least one or more pathologies belonging to a specific group), as shown in Table 1. For the cardiovascular and musculoskeletal diseases group, the cumulative effect $(0,1,2,>2)$ of more coexistent diseases was analysed. Finally, the comorbidities - that is the presence of two or more coexistent diseases - were analysed. 
Table 1 List of the investigated variables

\begin{tabular}{|c|c|}
\hline Diseases or group of diseases (ICDIX-CM code) & Therapeutic groups (ATC code) \\
\hline 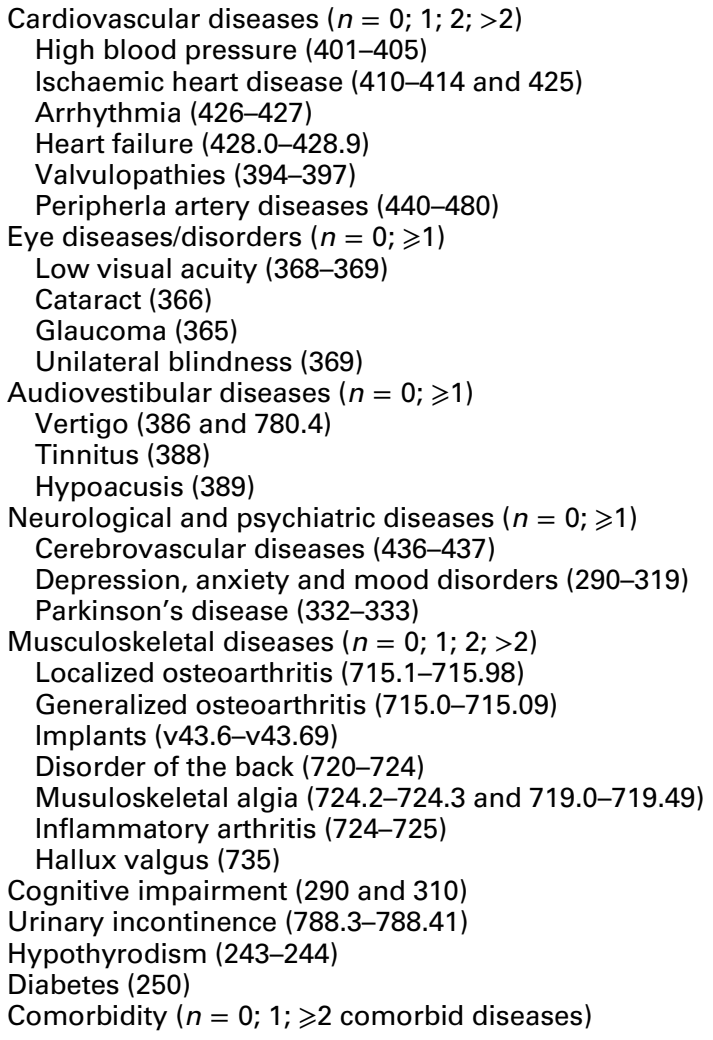 & $\begin{array}{l}\text { Antithrombotic agents (B01) } \\
\text { Cardiovascular drugs }(n=0 ; 1 ; \geqslant 2) \\
\text { Antihypertensives (C02) } \\
\text { Diuretics (C03) } \\
\text { Peripheral vasodilators (C04) } \\
\text { Beta blocking agents (C07) } \\
\text { Calcium channel blockers (C08) } \\
\text { Agents acting on the RAA system (C09) } \\
\text { Agents acting on musculo-skeletal system (M) } \\
\text { Analgesics (N02) } \\
\text { Antipsychotics (N05A) } \\
\text { Anxiolytics (N05B) } \\
\text { Hypnotics and sedatives (N05C) } \\
\text { Number of drugs }(0 ; 1 ; 2-3 ;>3)\end{array}$ \\
\hline
\end{tabular}

ATC $=$ anatomical therapeutic chemical; RAA $=$ renin-angiotensin-aldosterone.

The following medications (along with ATC code) were considered: antithrombotic agents (B01); antihypertensives (C02); diuretics (C03); peripheral vasodilators $(\mathrm{C} 04) ; \beta$ blocking agents (C07); calcium channel blockers (C08); agents acting on the renin-angiotensin-aldosterone (RAA) system (C09); agents acting on musculoskeletal system (M) (eg, muscle relaxants, vitamin D); analgesics (N02); antipsychotics (N05A); anxiolytics (N05B); and hypnotics and sedatives (N05C). The above variables were considered as dichotomous (presence or absence in the therapy of the subject). A variable was created to consider the number of cardiovascular drugs used in the therapy of each patient $(0,1, \geqslant 2)$. To assess the role of the number of drugs, a variable was created in order to take into account monopharmacy and polypharmacy.
Although there is no uniform definition of polypharmacy, we have defined it - in accordance with the literature on falls among the elderly - as the use of four or more medications (Leipzig et al., 1999a; 1999b; Ziere et al., 2006; Buatois et al., 2010).

A descriptive analysis with $\chi^{2}$ test for categorical and ordinal data and unpaired $t$-test for continuous data were performed in order to evaluate significant associations between falls and all the variables considered. The variables statistically associated with falls were entered into a multivariable logistic regression model conducted using the backward stepwise method. For each analysis, an $\alpha$ level of 0.05 is considered as significant. The statistical software IBM SPSS Statistics 20 and Stata 11 were used for data analyses.

Primary Health Care Research \& Development 2018; 19: 131-139 


\section{Vieri Lastrucci et al.}

\section{Results}

The sample consisted of 1220 subjects, of whom $43.1 \%$ were women and $56.9 \%$ men. The mean age was significantly different for men and women (women: $77.8 \pm 8.7$ years; men: $74.9 \pm 7.3$ years; $P<0.01)$. Of this sample, 142 subjects $(11.6 \%)$ had experienced one or more falls in the previous year. No significant dependence of the prevalence of falls on gender was observed. The prevalence of falls was found to significantly increase with age $(P=0.013)$.

The presence of coexistent risk factors for falls was found to increase with age; only 127 subjects had two or more risk factors in the age group 75 years or younger, whereas 506 subjects showed this feature in the age group 76 years or older (data not shown).

The medical conditions significantly associated with falls in the univariate analysis were: cognitive impairment; urinary incontinence; the presence of one or more audiovestibular diseases; the presence of one or more eye diseases, and the presence of one or more musculoskeletal diseases (see Table 2). Among the musculoskeletal, audiovestibular, and eye diseases, the following diseases/conditions were individually associated with falls: generalized osteoarthritis; the presence of implants, tinnitus, low visual acuity (LVA), and cataract. Furthermore, the risk of falling was found to increase significantly with the simultaneous presence of two or more of all the considered diseases (comorbid diseases).

In regard to medications, the following drugs were significantly associated with falls in the univariate analysis (see Table 2): peripheral vasodilators; agents acting on the RAA system, analgesics, and the use of two or more cardiovascular drugs. The total number of drugs present in therapy (polypharmacy) was not significantly associated with falls.

The variables statistically associated with falls in the univariate analysis were included in the multivariable regression model. Since the number of cardiovascular drugs is correlated with the use of peripheral vasodilators and of agents acting on the RAA system, the number of cardiovascular drugs

Table 2 Variables significantly associated with falls in the univariate analysis $\left(\chi^{2}\right.$ test $\left.P<0.05\right)$

\begin{tabular}{|c|c|c|c|}
\hline Variables & $\begin{array}{l}\text { Number of subjects } \\
\text { (\% of the sample) }\end{array}$ & $\begin{array}{l}\text { Falls prevalence } \\
\text { (number of fallers) }\end{array}$ & $P$ value \\
\hline Cognitive impairment & $47(3.9 \%)$ & $38.3 \%(18)$ & $<0.0001$ \\
\hline Urinary incontinence & $25(2 \%)$ & $28 \%(7)$ & 0.01 \\
\hline Audiovestibular diseases & $110(9 \%)$ & $18.2 \%(20)$ & 0.025 \\
\hline Eye diseases & $309(25.3 \%)$ & $15.5 \%(48)$ & 0.013 \\
\hline Tinnitus & $12(1 \%)$ & $41.7 \%(5)$ & 0.001 \\
\hline Cataract & $193(15.8 \%)$ & $16.6 \%(32)$ & 0.02 \\
\hline Low visual acuity & $46(3.8 \%)$ & $21.7 \%(10)$ & 0.029 \\
\hline Generalized osteoarthritis & $117(9.6 \%)$ & $22.2 \%(26)$ & $<0.0001$ \\
\hline Implants & $77(6.3 \%)$ & $21.1 \%(16)$ & 0.01 \\
\hline Musculoskeletal diseases & & & 0.019 \\
\hline 0 & $564(46.2 \%)$ & $8.5 \%(48)$ & \\
\hline 1 & $413(33.9 \%)$ & $14.3 \%(59)$ & \\
\hline 2 & $200(16.4 \%)$ & $14.5 \%(29)$ & \\
\hline$>2$ & $43(3.5 \%)$ & $14 \%(6)$ & \\
\hline Comorbid diseases & & & 0.002 \\
\hline 0 & $123(10.1 \%)$ & $0.2 \%(3)$ & \\
\hline 1 & $154(12.6 \%)$ & $0.5 \%(6)$ & \\
\hline$\geqslant 2$ & $943(77.3 \%)$ & $14.1 \%(133)$ & \\
\hline Peripheral vasodilators & $70(5.7 \%)$ & $20 \%(14)$ & 0.025 \\
\hline Agents acting on the RAA system & $501(41.0 \%)$ & $9.6 \%(48)$ & 0.049 \\
\hline Analgesics & $147(12 \%)$ & $18.4 \%(27)$ & 0.007 \\
\hline Cardiovascular drugs & & & 0.036 \\
\hline 0 & $375(30.7 \%)$ & $11.2 \%(42)$ & \\
\hline 1 & $701(57.5 \%)$ & $10.6 \%(74)$ & \\
\hline$\geqslant 2$ & $144(11.8 \%)$ & $18.1 \%(26)$ & \\
\hline
\end{tabular}

RAA = renin-angiotensin-aldosterone. 
Table 3 Multivariable logistic regression analysis

\begin{tabular}{llrl}
\hline Variables & OR & $P$ & $95 \% \mathrm{Cl}$ \\
\hline Age & 1.03 & 0.011 & $1.01-1.05$ \\
Gender (ref = male) & 1.12 & 0.540 & $0.77-1.63$ \\
Generalized osteoarthritis & 2.01 & 0.006 & $1.23-3.30$ \\
Tinnitus & 4.14 & 0.020 & $1.25-13.74$ \\
Cognitive impairment & 4.12 & $<0.001$ & $2.18-7.80$ \\
Comorbid diseases (ref $=0)$ & & & \\
$\quad 1$ & 1.64 & 0.500 & $0.40-6.73$ \\
$\quad 2$ & 5.40 & 0.005 & $1.68-17.39$ \\
\hline
\end{tabular}

Number of observations $=1220$; age was analysed as a continuous variable. The odds ratio (OR) indicates the annual increase in risk.

was dropped. In the multivariable logistic regression analysis (see Table 3), the variables that significantly remain associated with falls were found to be the following: age, generalized osteoarthritis, cognitive impairment, tinnitus, and two or more comorbid diseases. In particular, the risk of falling increases with age by about $3 \%$ annually $(P=0.011)$ and falls occur with a frequency that is approximately doubled in the presence of generalized osteoarthritis $(\mathrm{OR}=2.01$, $P=0.006)$. The presence of tinnitus, cognitive deficits, or two or more comorbid diseases resulted in an increase in the risk of falling by more than four times $(\mathrm{OR}=4.14, \quad P=0.02 ; \quad \mathrm{OR}=4.12$, $P<0.001$; and $\mathrm{OR}=5.4, P=0.005$, respectively).

\section{Discussion}

Our study analyses several heterogeneous fall predictors described in the literature (Graafmans et al., 1996; Rubenstein, 2006; Ambrose et al., 2013; Deandrea et al., 2013). According to the multivariable logistic regression analysis, the fall predictors detectable from routine medical records are the following factors: age, generalized osteoarthritis, cognitive impairment, tinnitus, and the presence of two or more comorbid diseases.

The analysis of fall predictors from routine medical records may allow us to identify which of the several known and hypothesized risk factors often determined through prospective and ad hoc studies - may be more relevant for developing quick and efficient tools for a preliminary fall-risk assessment in the context of proactive primary healthcare. However, it should be pointed out that the analysis of the routine medical records per se entails a retrospective investigation. This kind of study design is affected by a higher rate of underreported, under-detected, and inadequately evaluated falls (Rubenstein et al., 2004; Stevens et al., 2012). The capture-recapture method has been proposed as a means of improving and estimating the completeness of case ascertainment (Schootman et al., 2000); however, due to the unavailability of other sources of information in the study area, it was not possible to adopt this approach for the present study. These issues may eventually have led to an underestimation of fall incidence and risk factors in the sample.

The main limitation of the present study is related to the choice of including only one Community Health Center located in a rural municipality and to the modality used to select it (convenience criteria instead of random selection). Considering these aspects, the results of our study cannot be considered to be representative of urban areas and their generalizability to the general population should be confirmed by other studies. Since the municipality is served only by the selected Community Health Center, the reality of a whole medium-sized municipality could be considered to be well-represented in the study, and this together with the sample size and the consideration of various confounding factors - should be highlighted as the strength of the present study.

Some studies reported an increased risk in falls and fractures during the colder seasons due to slippage on ice and snow (Bulajic-Kopjar, 2000; Al-Azzani et al., 2016). In the present study, however, this seasonal effect was not taken into account, as the climate of the study area is characterized by mild winters. Hence, ice or snow cannot be assumed to be an extrinsic factor influencing falls incidence.

In our study, only $11.6 \%$ of the sample reported one or more falls in the previous year. This incidence is probably an underestimation, considering the retrospective nature of the study and its reliance on routine medical records. In fact, data from prospective studies indicate that each year about a third of the community-dwelling elderly population experience a fall (Hausdorff et al., 2001; Stalenhoef et al., 2002). This result shows that falls are still a major health issue that is hardly investigated by GPs. However, it should be taken into account that the low incidence may also be 
influenced by the fact that the sample of the study is limited to active elderly community dwellers, thus excluding people suffering from extremely debilitating diseases.

Among the variables investigated by the present study, some are well-established risk factors for falls (ie, age, cognitive impairment, generalized osteoarthritis, Parkinson's disease, vertigo, LVA, and psychotropic drugs), others have an unclear association with falls (ie, gender, cardiovascular drugs, and diabetes) and others have received little attention in the literature (tinnitus and multimorbidity).

Our study shows that in the routine medical records, some well-established risk factors for falls are found to be significantly associated with falls, while others are not. On the one hand - in line with the literature - age, cognitive impairment, and generalized osteoarthritis were found to be predictors of falls in the routine medical records. Indeed, in the literature a steadily rise of the rate of falls with age has been repeatedly described (Linattiniemi et al., 2009; Ambrose et al., 2013). Furthermore, cognitive impairment has been described as one of the most important risk factors for falls in the elderly (Muir et al., 2012); even minimal decrements in the Mini-Mental State Examination have been reported to increase the rate of falls (Gleason et al., 2009). Several studies have linked alterations in cognitive function to postural instability and gait disturbance (Brauer et al., 2001; Yogev-Seligmann et al., 2008; Yarnall et al., 2011). Finally, generalized osteoarthritis is usually associated with pain, functional impairment, and adverse psychological effects and hence could lead to an increased risk of falls among elderly people ( $\mathrm{Ng}$ and $\mathrm{Tan}, 2013)$.

On the other hand, although the literature identifies Parkinson's disease (Ambrose et al., 2006; Yarnall et al., 2011; Jacobs et al., 2014; Lubomski et al., 2015), LVA (Klein et al., 2003; De Boer et al., 2004), vertigo (Rubenstein et al., 1994; Tinetti et al., 1995; Nguyen et al., 2005; Pluijm et al., 2006; Gaßmann et al., 2009; Deandrea et al., 2010), and psychotropic drugs (Hartikainen et al., 2007; Woolcott et al., 2009) as risk factors for falls, they were not found to predict the risk of falls in the routine medical records. As for Parkinson's disease, the community-dwelling population along with the underestimation of the incidence of falls in the routine medical records - could explain the lack of association in the routine medical records to some extent. As far as LVA and vertigo are concerned, our findings may be influenced by the low prevalence of LVA and vertigo in the sample (3.8 and 3.2\%, respectively). In the literature, LVA and vertigo have been described to be present in around 10 and $30 \%$ of the elderly, respectively (Colledge et al., 1994; Vitale et al., 2006). As symptoms may be relatively mild or may progress slowly - or there may be the simultaneous presence of another disease (such as cognitive impairment) - elderly patients with LVA or vertigo may be unaware of or may underreport their symptoms, if these are not directly investigated (Oghalai et al., 2000; Chou et al., 2009). Moreover, in the context of community and family medicine, these fall predictors tend to be inadequately assessed and recorded, even for people with a reported history of falling (Rubenstein et al., 2004). Finally, regarding falls and the use of psychotropic drugs, a possible explanation of our results could lie in the fact that our sample was drawn from community-dwelling population, for whom the dosages and types of drugs used are usually milder than in the hospital or residential care populations; therefore, the occurrences of side effects that lead to falls (eg, hypotension, hypoglycaemia, drowsiness, abnormal reflexes, etc.) could be less frequent, and thus, in terms of routine medical records, these drugs may be less relevant predictors. However, more specific studies that take into account the dosages and the duration of therapy are needed for assessing the real effect of each psychotropic drug on falls in an active population of elderly community dwellers.

Despite the low prevalence of tinnitus in the sample, the presence of tinnitus was found to be associated with a higher risk of falling. This association is reported in literature (Homann et al., 2013), although it is inadequately investigated. Tinnitus is often associated with vertigo and vestibular disorders; among patients with Meniere's disease (a chronic vestibular disease common in the elderly), those with tinnitus are reported to be at a higher risk of falling (Kentala et al., 2001). Tinnitus may be an indicator of increased risk of falling in patients with vestibular disorders, and it may be more accurate than vertigo in predicting the risk of falls from the routine medical records. However, more specific studies are needed to draw a more definitive conclusion about this risk factor. 
In our study, multi-morbidity was found to be a significant predictor of falls. This result is in line with what has been reported in the literature (Faulkner et al., 2009; Shumway-Cook et al., 2009; Sibley et al., 2014). However, it should be noted that the association between multi-morbidity and falls has hardly been investigated to date. Since the prevalence of two of more co-existing chronic diseases in the elderly is increasing and the older population is becoming more and more complex (Marengoni et al., 2001), further studies are needed to better identify the specific weight of each comorbid disease in causing falls, in order to provide a more systematic and effective approach.

\section{Conclusions}

Falls are an important health issue that are still inadequately investigated and detected by GPs. Several fall predictors have been reported in the literature; the role played by each one has to be weighted accurately according to the characteristics of the examined population. In the context of proactive primary healthcare, the analysis of fall predictors from routine medical records may allow the determination of which of the several known and hypothesized risk factors may be more relevant to develop quick and efficient tools for a preliminary fall-risk assessment. The present study shows that it is already possible to detect patients at a risk of falling by using only data from the GPs' current medical records, without adding extra workload on the health personnel. A populationbased retrospective analysis of falls based on the GPs' routine medical records is more likely to be affected by the under-reporting of falls by the patients and by the under-detecting and inadequate evaluation of falls by GPs. This may, to some extent, explain the absence of significance for some well-established risk factors, which may result less suitable and appropriate for the development of a time- and cost-effective fall-risk assessment tools for the context of proactive primary healthcare.

Further larger studies are needed to better define a fall-risk assessment tool from routine medical records, and prospective studies will be necessary to evaluate and improve the reliability and validity of the so-developed screening tool.

\section{Acknowledgements}

The authors would like to thank the General Practioners of the Community Health Center "Casa della Salute Bucine" who supported this study.

\section{Financial Support}

This research received no specific grant from any funding agency, commercial or not-for-profit sectors.

\section{Conflicts of Interest}

None.

\section{Ethical Standards}

The authors assert that all procedures contributing to this work comply with the ethical standards of the relevant national and institutional guidelines on human experimentation (Local ethical committee) and with the Helsinki Declaration of 1975 , as revised in 2008.

\section{References}

Al-Azzani, W., Mak, D.A.M., Hodgson, P. and Williams, R. 2016: Epidemic of fractures during a period of snow and ice: has anything changed 33 years on? BMJ Open 6, e010582.

Ambrose, A., Levalley, A. and Verghese, J. 2006: A comparison of community-residing older adults with frontal and parkinsonian gaits. Journal of the Neurological Sciences 248, 215-18.

Ambrose, A., Paul, G. and Hausdorff, J.M. 2013: Risk factors for falls among older adults: a review of the literature. Maturitas 75, 51-61.

Brauer, S.G., Woollacott, M. and Shumway-Cook, A. 2001: The interacting effects of cognitive demand and recovery of postural stability in balance impaired elderly persons. Journals of Gerontology Series A: Biological Sciences and Medical Sciences 56, 489-96.

Buatois, S., Perret-Guillaume, C., Gueguen, R., Miget, P., Vançon, G., Perrin, P. and Benetos, A. 2010: A simple clinical scale to stratify risk of recurrent falls in communitydwelling adults aged 65 years and older. Physical Therapy 90,550 .

Bulajic-Kopjar, M. 2000: Seasonal variations in incidence of fractures among elderly people. Injury Prevention 6, 16-19.

Chou, R., Dana, T. and Bougatsos, C. 2009: Screening older adults for impaired visual acuity: a review of the evidence for the U.S. Preventive Services Task Force. Annals of Internal Medicine 151, 44-58. 
Colledge, N.R., Wilson, J.A., Macintyre, C.C. and MacLennan, W.J. 1994: The prevalence and characteristics of dizziness in an elderly community. Age and Ageing 23, 117-20.

De Boer, M.R., Pluijm, S.M., Lips, P., Moll, A.C., VölkerDieben, H.J., Deeg, D.J. and van Rens, G.H. 2004: Different aspects of visual impairment as risk factors for falls and fractures in older men and women. Journal of Bone and Mineral Research 19, 1539-47.

Deandrea, S., Bravi, F., Turati, F., Lucenteforte, E., La Vecchia, C. and Negri, E. 2013: Risk factors for falls in older people in nursing homes and hospitals. A systematic review and meta-analysis. Archives of Gerontology and Geriatrics 56, 407-15.

Deandrea, S., Lucenteforte, E., Bravi, F., Foschi, R., La Vecchia, C. and Negri, E. 2010: Risk factors for falls in community-dwelling older people: a systematic review and meta-analysis. Epidemiology 21, 658-68.

Faulkner, K.A., Cauley, J.A., Studenski, S.A., Landsittel, D.P., Cummings, S.R., Ensrud, K.E., Donaldson, M.G. and Nevitt, M.C. 2009: Lifestyle predicts falls independent of physical risk factors. Osteoporosis International 20, 2025-34.

Fried, L.P., Ferrucci, L., Darer, J., Williamson, J.D. and Anderson, G. 2004: Untangling the concepts of disability, frailty, and comorbidity: implications for improved targeting and care. Journals of Gerontology. Series A: Biological and Medical Sciences 59, 255-63.

Gaßmann, K.G., Rupprecht, R. and Freiberger, E. 2009: Predictors for occasional and recurrent falls in communitydwelling older people. Zeitschrift für Gerontologie und Geriatrie 42, 3-10.

Gleason, C.E., Gangnon, R.E., Fischer, B.L. and Mahoney, J.E. 2009: Increased risk for falling associated with subtle cognitive impairment: secondary analysis of a randomized clinical trial. Dementia and Geriatric Cognitive Disorders 27, 557-63.

Goldacre, M.J., Roberts, S.E. and Yeates, D. 2002: Mortality after admission to hospital with fractured neck of femur: database study. British Medical Journal 325, 868-869.

Graafmans, W.C., Ooms, M.E., Hofstee, H.M.A., Bezemer, P.D., Bouter, L.M. and Lips, P. 1996: Falls in the elderly: a prospective study of risk factors and risk profiles. American Journal of Epidemiology 143, 1129-36.

Hartikainen, S., Lonnroos, E. and Louhivuori, K. 2007: Medication as a risk factor for falls: critical systematic review. Journal of Gerontology: Medical Sciences 62, 1172-81.

Hausdorff, J.M., Rios, D.A. and Edelberg, H.K. 2001: Gait variability and fall risk incommunity-living older adults: a 1-year prospective study. Archives of Physical Medicine and Rehabilitation 82, 1050-56.

Homann, B., Plaschg, A., Grundner, M., Haubenhofer, A., Griedl, T., Ivanic, G., Hofer, E., Fazekas, F. and Homann, C.N. 2013: The impact of neurological disorders on the risk for falls in the community dwelling elderly: a case-controlled study. British Medical Journal Open 3, e003367.

Primary Health Care Research \& Development 2018; 19: 131-139
Jacobs, J.V., Nutt, J.G., Carlson-Kuhta, P., Allen, R. and Horak, F.B. 2014: Dual tasking during postural stepping responses increases falls but not freezing in people with Parkinson's disease. Parkinsonism \& Related Disorders 20, 779-81.

Kentala, E., Havia, M. and Pyykkö, I. 2001: Short-lasting drop attacks in Meniere's disease. Otolaryngology-Head and Neck Surgery 124, 526-30.

Klein, B.E., Moss, S.E., Klein, R., Lee, K.E. and Cruickshanks, K.J. 2003: Associations of visual function with physical outcomes and limitations 5 years later in an older population: the Beaver Dam Eye Study. Ophthalmology 110, 644-50.

Leipzig, R.M., Cumming, R.G. and Tinetti, M.E. 1999a: Drugs and falls in older people: a systematic review and metaanalysis: I. Psychotropic drugs. Journal of the American Geriatrics Society 47, 30-39.

Leipzig, R.M., Cumming, R.G. and Tinetti, M.E. 1999b: Drugs and falls in older people: a systematic review and metaanalysis: II. Cardiac and analgesic drugs. Journal of the American Geriatrics Society 47, 40-50.

Linattiniemi, S., Jokelainen, J. and Luukinen, H. 2009: Falls risk among a very old home-dwelling population. Scandinavian Journal of Primary Health Care 27, 25-30.

Lord, S.R., Menz, H.B. and Sherrington, C. 2006: Home environment risk factors for falls in older people and the efficacy of home modifications. Age and Ageing 35 (Suppl 2), ii55-ii59.

Lubomski, M., Rushworth, R.L. and Tisch, S. 2015: Hospitalisation and comorbidities in Parkinson's disease: a large Australian retrospective study. Journal of Neurology Neurosurgery \& Psychiatry 86, 324-30.

Marengoni, A., Angleman, S., Melis, R., Mangialasche, F., Karp, A., Garmen, A., Meinow, B. and Fratiglioni, L. 2001: Aging with multimorbidity: a systematic review of the literature. Ageing Research Reviews 10, 430-39.

Muir, S.W., Gopaul, K. and Montero Odasso, M.M. 2012: The role of cognitive impairment in fall risk among older adults: a systematic review and meta-analysis. Age and Ageing 41, 299-308.

Murphy, J. and Isaacs, B. 1982: The post - fall syndrome. A study of 36 elderly fallers. Gerontology 28, 265-70.

Ng, C.T. and Tan, M.P. 2013: Osteoarthritis and falls in the older person. Age and Ageing 42, 561-66.

Nguyen, N.D., Pongchaiyakul, C., Center, J.R., Eisman, J.A. and Nguyen, T.V. 2005: Identification of high-risk individuals for hip fracture: a 14-year prospective study. Journal of Bone and Mineral Research 20, 1921-28.

Oghalai, J.S., Manolidis, S., Barth, J.L., Stewart, M.G. and Jenkins, H.A. 2000: Unrecognized benign paroxysmal positional vertigo in elderly patients. Otolaryngology-Head and Neck Surgery 122, 630-34.

Pluijm, S.M., Smit, J.H., Tromp, E.A., Stel, V.S., Deeg, D.J., Bouter, L.M. and Lips, P. 2006: A risk profile for identifying community-dwelling elderly with a high risk of recurrent falling: results of a 3-year prospective study. Osteoporosis International 17, 417-25. 
Rubenstein, L.Z. 2006: Falls in older people: epidemiology, risk factors and strategies for prevention. Age and Aging 35 (Suppl 2), ii37-ii41.

Rubenstein, L.Z. and Josephson, K.R. 2002: The epidemiology of falls and syncope. Clinics in Geriatric Medicine 18, 141-58.

Rubenstein, L.Z., Josephson, K.R. and Robbins, A.S. 1994: Falls in the nursing home. Annals of Internal Medicine 121, 442-51.

Rubenstein, L.Z., Solomon, D.H., Roth, C.P., Young, R.T., Shekelle, P.G., Chang, J.T., MacLean, C.H., Kamberg, C.J., Saliba, D. and Wenger, N.S. 2004: Detection and management of falls and instability in vulnerable elders by community physicians. Journal of the American Geriatrics Society 52, 1527-31.

Schootman, M., Harlan, M. and Fuortes, L. 2000: Use of the capture-recapture method to estimate severe traumatic brain injury rates. The Journal of Trauma and Acute Care Surgery 48, 70-75.

Shumway-Cook, A., Ciol, M.A., Hoffman, J., Dudgeon, B.J., Yorkston, K. and Chan, L. 2009: Falls in the medicare population: incidence, associated factors, and impact on health care. Physical Therapy 89, 324-32.

Sibley, K.M., Voth, J., Munce, S.E., Straus, S.E. and Jaglal, S.B. 2014: Chronic disease and falls in community-dwelling Canadians over 65 years old: a population-based study exploring associations with number and pattern of chronic conditions. BMC Geriatrics 14, 22.

Stalenhoef, P.A., Diederiks, J.P.M., Knottnerus, J.A., Kester, A.D.M. and Crebolder, H.F.J.M. 2002: A risk model for the prediction of recurrent falls in community-dwelling elderly: a prospective cohort study. Journal of Clinical Epidemiology 55, 1088-94.

Stevens, J.A., Ballesteros, M.F., Mack, K.A., Rudd, R.A., DeCaro, E. and Adler, G. 2012: Gender differences in seeking care for falls in the aged medicare population. American Journal of Preventive Medicine 43, 59-62.

Tinetti, M.E., Doucette, J., Claus, E. and Marottoli, R. 1995: Risk factors for serious injury during falls by older persons in the community. Journal of the American Geriatrics Society 43, $1214-21$.
Tinetti, M.E., Gordon, C., Sogolow, E., Lapin, P. and Bradley, E.H. 2006: Fall-risk evaluation and management: challenges in adopting geriatric care practices. The Gerontologist 46, $717-25$.

Todd, C. and Skelton, D. 2004: What are the main risk factors for falls amongst older people and what are the most effective interventions to prevent these falls? Health Evidence Network report. Copenhagen: World Health Organization Regional Office for Europe.

Vitale, S., Cotch, M.F. and Sperduto, R.D. 2006: Prevalence of visual impairment in the United States. JAMA 295, 2158-63.

Wenger, N.S., Solomon, D.H., Roth, C.P., MacLean, C.H., Saliba, D., Kamberg, C.J., Rubenstein, L.Z., Young, R.T., Sloss, E.M., Louie, R., Adams, J., Chang, J.T., Venus, P.J., Schnelle, J.F. and Shekelle, P.G. 2003: The quality of medical care provided to vulnerable community-living older patients. Annals of Internal Medicine 139, 740-47.

Woolcott, J.C., Richardson, K.J., Wiens, M.O., Patel, B., Marin, J., Khan, K.M. and Marra, C.A. 2009: Metaanalysis of the impact of 9 medication classes on falls in elderly persons. Archives of Internal Medicine 169, 1952-60.

Yarnall, A., Rochester, L. and Burn, D.J. 2011: The interplay of cholinergic function, attention, and falls in Parkinson's disease. Movement Disorders 26, 2496-503.

Yogev-Seligmann, G., Hausdorff, J.M. and Giladi, N. 2008: The role of executive function and attention in gait. Movement Disorders 23, 329-42.

Ziere, G., Dieleman, J. P., Hofman, A., Pols, H.A., Van Der Cammen, T.J.M. and Stricker, B.H. 2006: Polypharmacy and falls in the middle age and elderly population. British Journal of Clinical Pharmacology 61, 218-23.

Zijlstra, G.A., van Haastregt, J.C., van Eijk, J.T., van Rossum, E., Stalenhoef, P.A. and Kempen, G.I. 2007: Prevalence and correlates of fear of falling, and associated avoidance of activity in the general population of community-living older people. Age and Ageing 36, 304-9. 